\title{
Minimally invasive removal of an infected Edwards S3 transcatheter aortic valve
}

\author{
Tom C. Nguyen, MD, FACS, FACC, ${ }^{a}$ Robert D. Rice, MD, ${ }^{\mathrm{b}}$ Juan B. Umana-Pizano, MD, ${ }^{\mathrm{a}}$ and \\ Pranav Loyalka, MD, ${ }^{\mathrm{c}}$ Houston, Tex, and Fort Gordon, Ga
}

\footnotetext{
From the ${ }^{\mathrm{a}}$ Department of Cardiothoracic and Vascular Surgery, and ${ }^{\mathrm{c}}$ Division of Cardiology, Department of Internal Medicine, University of Texas Health Science Center Houston, McGovern Medical School, Memorial Hermann Hospital-Heart and Vascular Institute, Houston, Tex; and ${ }^{\mathrm{b}}$ Department of Cardiothoracic Surgery, Dwight D. Eisenhower Army Medical Center, Fort Gordon, Ga.

Disclosures: T.C.N. is a consultant for Edwards Lifesciences, Abbott, and LivaNova outside the submitted work. All other authors have nothing to disclose with regard to commercial support.

No funding was provided for this report.

Received for publication April 20, 2018; revisions received May 18, 2018; accepted for publication June 5, 2018; available ahead of print July $6,2018$.

Address for reprints: Tom C. Nguyen, MD, FACS, FACC, 6400 Fannin St, Suite 2850, Houston, TX 77030 (E-mail: tom.c.nguyen@gmail.com).

J Thorac Cardiovasc Surg 2019;157:e113-6

$0022-5223 / \$ 0.00$

Published by Elsevier Inc. on behalf of The American Association for Thoracic Surgery

https://doi.org/10.1016/j.jtcvs.2018.06.001
}

$\square$ Video clip is available online.

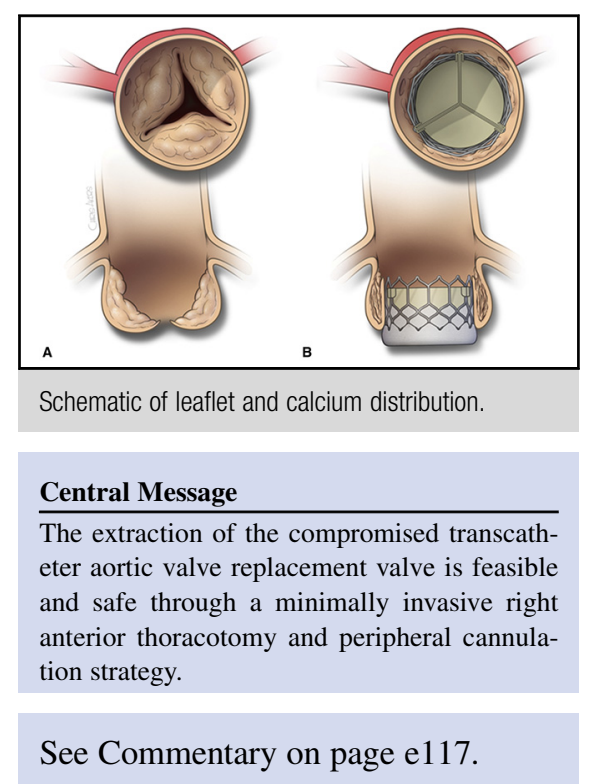

Transcatheter aortic valve replacement (TAVR) has rapidly advanced from its original role in high-risk population groups to those at intermediate and potentially even low risk. ${ }^{1}$ Recent data indicate that the volume of TAVRs conducted has now surpassed that of surgical aortic valve replacement, suggesting a paradigm shift in the treatment approach to severe aortic stenosis. ${ }^{2}$ Because of this increased volume, transcatheter heart valve (THV) removal may become increasingly more common, particularly as TAVR continues to be performed more frequently in younger, lower-risk patient cohorts.

In this case report, we describe a patient with THV infective endocarditis and the subsequent technique and unique findings of explanting the THV through a minimally invasive right thoracotomy.

\section{CASE}

An 81-year-old man with a history of chronic kidney disease, congestive heart failure, diabetes mellitus, permanent pacemaker, deep venous thrombosis with inferior vena cava filter placement, and prostate cancer underwent an uneventful TAVR in June 2016 (Society of Thoracic Surgeons risk score of $10.6 \%$ and annular area of $606.7 \mathrm{~mm}^{2}$; Edwards 29-mm S3 valve; Edwards Lifesciences Corp, Irvine, Calif). Approximately 2 years later, the patient presented with persistent Enterococcus faecalis bacteremia, despite long-term antibiotic therapy, and a 2.4-cm mobile mass attached to the previously placed THV. Treatment options were discussed at our multidisciplinary valve conference, resulting in a consensus to proceed with a minimally invasive right thoracotomy approach to remove the infected THV.

\section{DESCRIPTION OF OPERATIVE TECHNIQUE}

Approaches for a right anterior thoracotomy aortic valve replacement have been previously reported with a summary of our technique described here. The patient's left femoral vessels were exposed. A venous cannula was then placed into the common femoral vein with the Seldinger technique and advanced into the superior vena cava under echocardiographic and fluoroscopic guidance. Navigating the venous catheter was uneventful, despite the previous inferior vena cava filter (Figure 1). The femoral artery was accessed, but there was resistance when advancing the 0.035 -inch soft $\mathrm{J}$-wire into the iliac artery. Portable fluoroscopy revealed a tortuous iliac artery (Figure 1, A, 


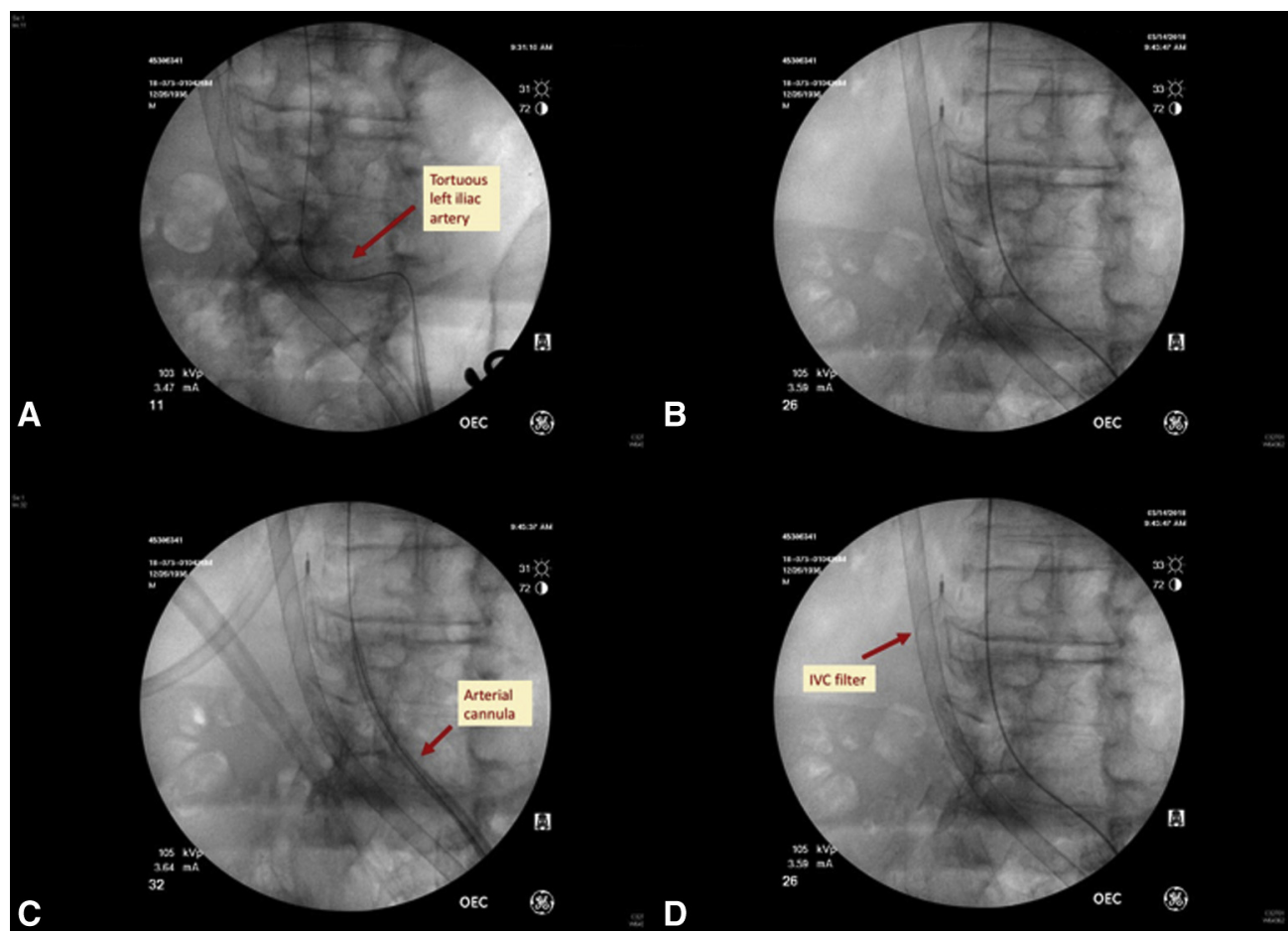

FIGURE 1. Fluoroscopic images of peripheral arterial cannulation; A, Tortuous left iliac artery with difficulty advancing the arterial cannula; B, Iliac artery straightened using a stiff Amplatz wire; C, Arterial cannula now advanced up the iliac with minimal resistance; and D, Peripheral venous cannulation through a previous inferior vena cava filter. $I V C$, Inferior vena cava

and Video 1). The 0.035-inch J-wire was exchanged for a glide wire, which was then advanced up the iliac under direct fluoroscopic guidance (Figure 1, B). A multipurpose

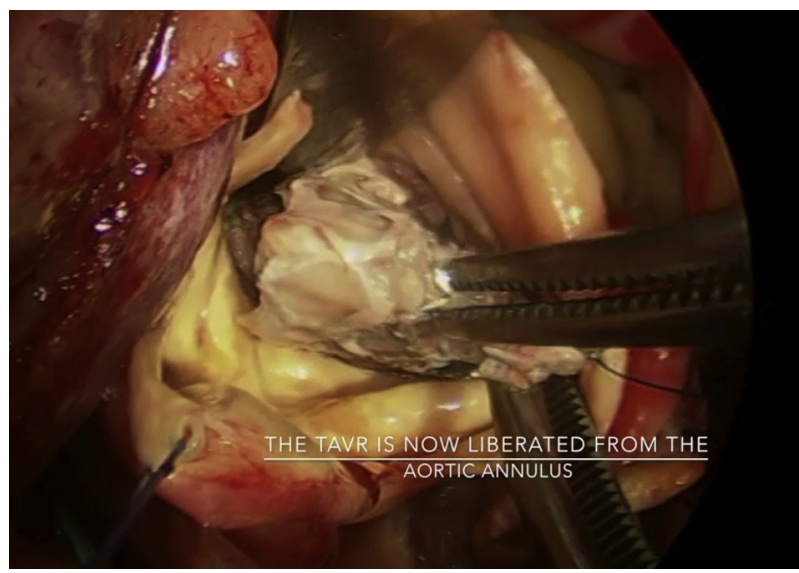

VIDEO 1. This video shows peripheral cannulation techniques for a tortuous iliac artery and previous inferior vena cava filter. The video also demonstrates the transcatheter heart valve (THV) firmly incorporated into the surrounding tissue and the techniques for its extraction. IVC, Inferior vena cava; TAVR, transcatheter aortic valve replacement. Video available at: https://www.jtcvs.org/article/S0022-5223(18)31552-6/fulltext. catheter was then advanced over the glide wire, and the glide wire was exchanged with an Amplatz stiff wire (Boston Scientific Corporation, Marlborough, Mass) to

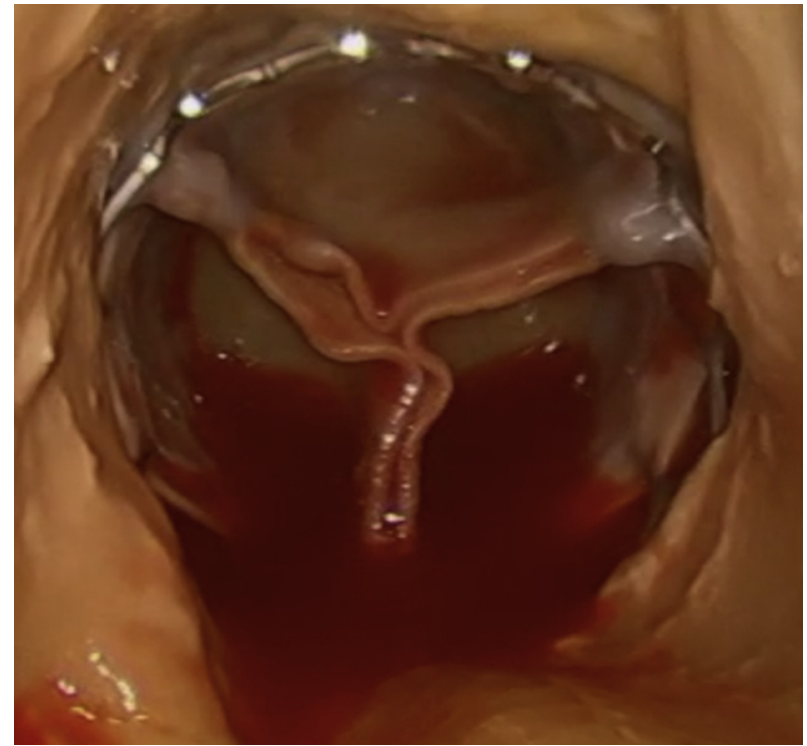

FIGURE 2. Direct view of the previously implanted transcatheter heart valve (THV). The THV is firmly adherent and incorporated into the surrounding tissue. Liberation of the valve ultimately required serial crushing of the THV. 


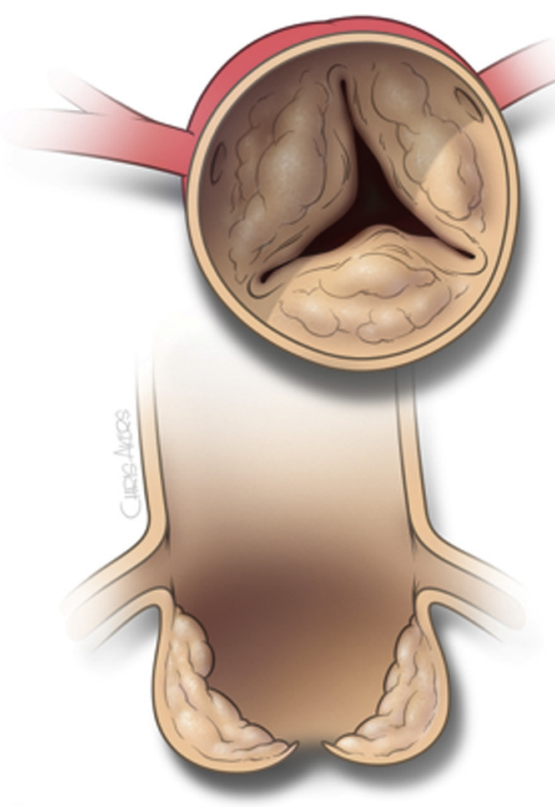

A

FIGURE 3. Schematic of leaflet and calcium distribution before and after transcatheter heart valve (THV) implantation; A, Demonstrates native calcified aortic leaflets; B, Demonstrates implanted THV. The native calcified aortic leaflets were crushed against the aortic wall and not visible on gross inspection.

straighten the iliac artery (Figure 1,C). This supplied an adequate rail to advance an arterial cannula without resistance. The patient was subsequently placed on peripheral cardiopulmonary bypass.

We then directed our attention to the right anterior chest incision through the third intercostal space and exposed the aorta. An inverted U-shaped aortotomy was then completed at level of the aortic fat pad providing an excellent view of the previously implanted THV (Figure 2). There were several noteworthy observations: (1) The previously implanted THV valve was firmly anchored into the aortic annulus and incorporated into the surrounding tissue, and (2) the native calcified aortic leaflets were not immediately visible and potentially inconsequential after TAVR implantation (Figures 2 and 3; and Video 1). It appeared that the native aortic valve leaflets and calcium were crushed between the THV and aortic wall. At first, we attempted to remove the THV by débriding the surrounding tissue, but it was clear that the S3 valve was firmly incorporated. Removing the valve required serial crushing of the frame with resection of native leaflet tissue. After removal of the THV, the vegetation was noted on the ventricular side of the THV (Figure 4). The remaining native valve leaflets were removed with sharp and rongeur dissection, and a 27-mm Edwards Magna Ease bioprosthetic valve (Edwards Lifesciences) was implanted in the standard fashion. The total cross-clamp and

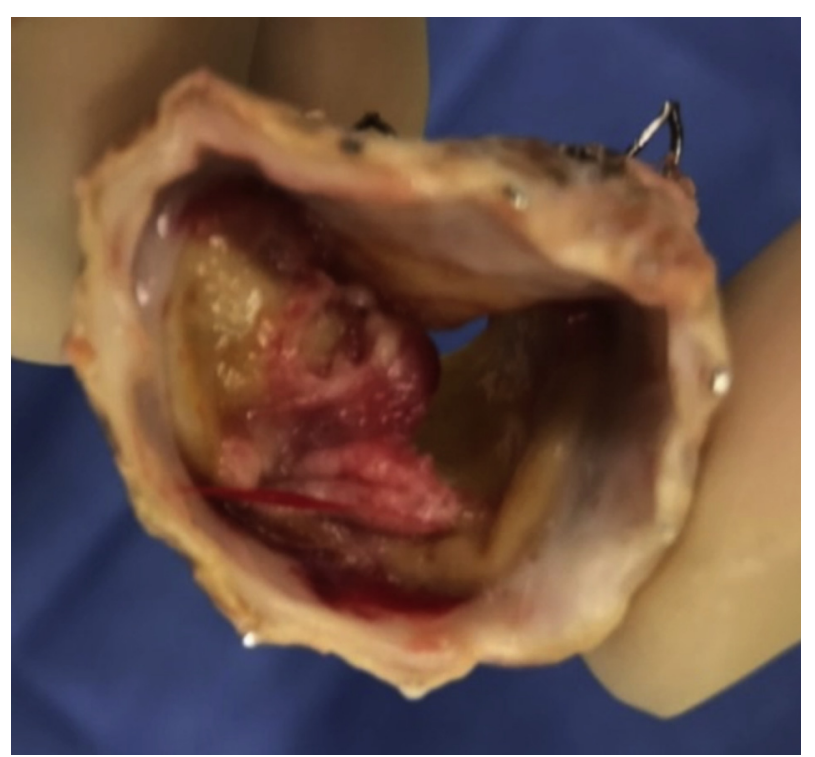

FIGURE 4. Vegetation on the ventricular side of the previously implanted transcatheter heart valve. 
cardiopulmonary bypass times were 75 minutes and 130 minutes, respectively.

\section{DISCUSSION}

TAVR is being used with increasing frequency in both high-risk and intermediate-risk populations. There may be a growing need for TAVR reoperation in the future, especially as the technology moves towards a younger, lower-risk cohort. ${ }^{3}$ Furthermore, Latib and colleagues ${ }^{4}$ report a $1 \%$ to $2 \%$ incidence of THV infective endocarditis, with a $60 \%$ mortality. ${ }^{4}$ It is therefore important for surgeons to understand potential techniques for liberating an implanted THV.

This case report describes the following findings: (1) Removal of a previously implanted THV was feasible through a minimally invasive right anterior thoracotomy by experienced minimally invasive surgeons. (2) The previously implanted THV was firmly incorporated into the surrounding aortic tissue and required serial crushing for extraction. (3) Finally, it is important for minimally invasive surgeons to be facile with endovascular techniques to potentially navigate tortuous vessels.

In patients with a previously implanted THV with either structural valve deterioration or infective endocarditis, minimally invasive reoperation may be considered contraindicated, particularly in high-risk patients. In contrast, we report that minimally invasive reoperation by experienced surgeons for removal of a THV is feasible and safe, with excellent visualization and acceptable crossclamp times (Figure 2 and Video 1). To account for all potential scenarios during explantation of a THV, minimally invasive valve surgeons must be prepared to perform advanced aortic root surgery. Furthermore, preoperative imaging is paramount to predict the need for more complex aortic reconstruction, and full sternotomy may be the preferred approach in such cases.

We also discovered that the previously implanted THV becomes firmly incorporated into the surrounding aortic tissue, requiring a stepwise approach for removal. It is important to note that extraction of a THV is different from extraction of a standard surgical aortic valve. After implantation, the THV becomes firmly incorporated into surrounding leaflet and aortic tissue. The transcatheter valve frame, on proper deployment, covers the calcified native aortic valve leaflets, which effectively holds the TAVR valve securely in place. The TAVR frame coverage of the native aortic valve tissue allows the left ventricular outflow tract to be unobstructed after proper insertion. Removal of the TAVR valve after incorporation requires stepwise serial folding of the frame of the valve onto itself to liberate the valve from the surrounding native valve tissue (Video 1). Care should be taken during these maneuvers to account for all pieces of the implanted valve and the valvular vegetation. After removal of the TAVR valve, standard surgical valve implantation can proceed in a standard fashion, with removal of the remaining native aortic leaflets and securing of the surgical valve to the aortic annulus.

Previous arguments contended that surgical aortic valve replacement affords a theoretic advantage relative to TAVR because the calcified aortic leaflets are surgically resected relative to TAVR, in which the native leaflets remain in situ. This case illustrates that the native calcified aortic leaflets were completely crushed against the aortic wall and did not obstruct the outflow tract (Figure 3 and Video 1). Moreover, the native aortic leaflets were not grossly visible around the THV frame. After liberation of the THV, the underlying native aortic leaflets were relatively soft and pliable, suggesting that there may be an element of native leaflet remodeling after THV implantation (Video 1). On the basis of these findings, we believe that the previously held argument that surgical aortic valve replacement affords an advantage relative to TAVR because leaflets are resected may not be warranted and that leaving native leaflets in situ may be inconsequential.

Finally, we believe that it is important for minimally invasive valve surgeons to be facile with basic endovascular skills for percutaneous cannulation in the advent of peripheral vascular anatomic variations. In this case, the tortuous iliac artery was effectively managed with a straightforward wire exchange with a multipurpose catheter for a stiff Amplatz wire to straighten the iliac and advance the arterial cannula. This case also highlights that patients with previous inferior vena cava filter placement are not ineligible for peripheral venous cannulation if catheter advancement is completed under fluoroscopic guidance.

\section{CONCLUSIONS}

THV infective endocarditis represents a complex problem with an associated high mortality. ${ }^{4}$ The extraction of the compromised TAVR valve is feasible and safe through a minimally invasive right anterior thoracotomy and peripheral cannulation strategy. The technique for removal of the incorporated THC requires a serial crushing in contrast to removal of a surgically implanted valve.

\section{References}

1. Terré JA, George I, Smith CR. Pros and cons of transcatheter aortic valve implantation (TAVI). Ann Cardiothorac Surg. 2017;6:444-52.

2. Nguyen TC, Terwelp MD, Thourani VH, Zhao Y, Ganim N, Hoffman C, et al. Clinical trends in surgical, minimally invasive and transcatheter aortic valve replacement. Eur J Cardiothorac Surg. 2017;51:1068-92.

3. Mazine A, Ouzounian M. Aortic valve replacement in young and middle-aged adults: looking beyond the tree that hides the forest. Ann Transl Med. 2017;5:92.

4. Latib A, Naim C, De Bonis M, Sinning JM, Maisano F, Barbanti M, et al. TAVR-associated prosthetic valve endocarditis: results of a large, multicenter registry. J Am Coll Cardiol. 2014;64:2176-8. 